\title{
ON THE CONNECTEDNESS OF SELF-AFFINE TILES
}

\author{
IBRAHIM KIRAT AND KA-SING LAU
}

\begin{abstract}
Let $T$ be a self-affine tile in $\mathbb{R}^{n}$ defined by an integral expanding matrix $A$ and a digit set $D$. The paper gives a necessary and sufficient condition for the connectedness of $T$. The condition can be checked algebraically via the characteristic polynomial of $A$. Through the use of this, it is shown that in $\mathbb{R}^{2}$, for any integral expanding matrix $A$, there exists a digit set $D$ such that the corresponding tile $T$ is connected. This answers a question of Bandt and Gelbrich. Some partial results for the higher-dimensional cases are also given.
\end{abstract}

\section{Introduction}

Throughout the paper, we use $M_{n}(\mathbb{Z})$ to denote the set of $n \times n$ matrices with entries in $\mathbb{Z}$, and $A$ is an expanding integral matrix in $M_{n}(\mathbb{Z})$, that is, all its eigenvalues have modulus $>1$. Let $|\operatorname{det} A|=q$ and let $D=\left\{d_{1}, \ldots, d_{q}\right\} \subseteq \mathbb{R}^{n}$ be a set of $q$ distinct vectors, called a $q$-digit set. If we let $S_{j}(x)=A^{-1}\left(x+d_{j}\right), 1 \leqslant j \leqslant q$, then they are contractive maps under a suitable norm in $\mathbb{R}^{n}[\mathbf{9}$, p. 29] and it is well known that there is a unique non-empty compact set $T$ satisfying $T=\bigcup_{j=1}^{q} S_{j}(T)[\mathbf{3}, \mathbf{8}]$, which is explicitly given by

$$
T:=T(A, D)=\left\{\sum_{i=1}^{\infty} A^{-i} d_{j_{i}}: d_{j_{i}} \in D\right\} .
$$

$T$ is called the attractor of the system $\left\{S_{j}\right\}_{j=1}^{q}$, and is called a self-affine tile if its Lebesgue measure $\mu(T)$ is positive. In particular, a self-affine tile $T(A, D)$ with $D \subseteq$ $\mathbb{Z}^{n}$ is called an integral self-affine tile. A simple sufficient condition for $\mu(T)>0$ is that $D$ is a complete set of coset representatives of $\mathbb{Z}^{n} / A \mathbb{Z}^{n}[\mathbf{1}]$.

One of the very interesting aspects of the self-affine tiles is the connectedness. This property has been considered by Bandt and Gelbrich [2], Gröchenig and Haas [5] and Hacon et al. [6]. So far, the knowledge is still very limited, and it is our main purpose in this paper to consider this property.

It is almost trivial to see that in $\mathbb{R}$, for $A=[q]$ and $D=\{0, d, \ldots,(q-1) d\}$ with $q, d \in \mathbb{Z}^{+}, T$ is an interval. The converse is also true (see Corollary 4.4). Hence it is natural to consider an extension of such tiles in $\mathbb{R}^{n}$ in regard to the connectedness. We give a criterion for such an attractor to be a tile by the following theorem.

Theorem 1.1. Suppose that $A \in M_{n}(\mathbb{Z})$ is an expanding matrix with $|\operatorname{det} A|=q$, where $q \geqslant 2$ is a prime. Let $D=\left\{d_{1} v, \ldots, d_{q} v\right\}$ with $v \in \mathbb{R}^{n} \backslash\{0\}$, for $d_{i} \in \mathbb{Z}$. Then $T$ is a self-affine tile (that is, $\mu(T)>0$ ) if and only if $\left\{v, A v, \ldots, A^{n-1} v\right\}$ is a linearly independent set and $\left\{d_{1}, \ldots, d_{q}\right\}=q^{l}\left\{d_{1}^{\prime}, \ldots, d_{q}^{\prime}\right\}$, where $l$ is a nonnegative integer with $\left\{d_{1}^{\prime}, \ldots, d_{q}^{\prime}\right\}$, is a complete set of coset representatives of $\mathbb{Z}_{q}$.

Received 6 November 1998; revised 11 March 1999.

2000 Mathematics Subject Classification 52C20, 52C22 (primary), 28A80 (secondary).

J. London Math. Soc. (2) 62 (2000) 291-304 
In particular, if $v \in \mathbb{Z}^{n} \backslash\{0\}$, then $\left\{v, A v, \ldots, A^{n-1} v\right\}$ is automatically a linearly independent set. Hence $T$ is a self-affine tile if and only if the above $\left\{d_{1}^{\prime}, \ldots, d_{q}^{\prime}\right\}$ is a complete set of coset representatives of $\mathbb{Z}_{q}$.

We call the digit set of the above form a collinear digit set. In Theorem 1.1, if $q$ is not a prime, then we need to assume additional conditions on $A$ and $v$ to ensure that $\mu(T)>0$ (Theorem 3.3). It follows easily from the theorem that if $|\operatorname{det} A|=2$, then for any 2 -digit set with integer entries, $T$ has positive measure. It is also connected, as was proved in [6].

Our approach to attack the connectedness is the following general criterion.

THEOREM 1.2. Let $T$ be an attractor defined by $A \in M_{n}(\mathbb{Z})$ and a digit set $D \subseteq \mathbb{R}^{n}$. Define

$$
\mathscr{E}=\left\{\left(d_{i}, d_{j}\right):\left(T+d_{i}\right) \cap\left(T+d_{j}\right) \neq \varnothing, d_{i}, d_{j} \in D\right\} .
$$

Then $T$ is connected if and only if for any pair of distinct $d_{i}, d_{j} \in D$, there exists a finite set $\left\{d_{j_{1}}, \ldots, d_{j_{k}}\right\} \subseteq D$ such that $d_{j_{1}}=d_{i}, d_{j_{k}}=d_{j}$ and $\left(d_{j_{l}}, d_{j_{l+1}}\right) \in \mathscr{E}$ for $1 \leqslant l \leqslant k-1$.

It is easy to check that $\left(d_{i}, d_{j}\right) \in \mathscr{E}$ if and only if

$$
d_{i}-d_{j}=\sum_{k=1}^{\infty} A^{-k} v_{k}, \quad v_{k} \in D-D .
$$

We show that in many cases the expression can be checked by using the characteristic polynomial of $A$ (Proposition 5.3). This, together with Theorem 1.1 and the other results in Section 3, leads us to the following theorem.

THEOREM 1.3. Let $A \in M_{2}(\mathbb{Z})$ be an expanding matrix with $|\operatorname{det} A|=q$ (not necessarily prime). Then there exists a digit set $D=\left\{d_{1}, \ldots, d_{q}\right\} \subseteq \mathbb{Z}^{2}$ such that $T$ is a connected tile.

This answers a question mentioned in [2] (see also [5]). By using a similar technique, we also obtain some partial results in $\mathbb{R}^{3}$. A more satisfactory solution in $\mathbb{R}^{n}$ depends on a conjecture on the characteristic polynomial of $A$, which we will discuss at the end of the paper.

We organize the paper as follows. In Section 2, we start with some elementary facts on the tiles, and an algebraic result on the irreducible polynomials. We prove Theorem 1.1 in Section 3, and the connectedness criterion, Theorem 1.2, in Section 4. The technique of checking the criterion is discussed in Section 5 (Proposition 5.3). By using this, we prove Theorem 1.3 and some partial results in higher dimensions. In Section 6, we make some concluding remarks and pose some open problems.

\section{Preliminaries}

Some of the results in this section are probably known. However, we will include the proofs for completeness. We begin with a simple result for the attractors.

Proposition 2.1. Let $A \in M_{n}(\mathbb{Z})$ be an expanding matrix with $|\operatorname{det} A|=q$, and let $D_{1}, D_{2}$ be two q-digit sets with $D_{2}=x_{0}+D_{1}$ for some $x_{0} \in \mathbb{R}^{n}$. Then the two attractors $T_{1}$ and $T_{2}$ corresponding to $\left(A, D_{1}\right)$ and $\left(A, D_{2}\right)$ are related by

$$
T_{2}=T_{1}+(A-I)^{-1} x_{0} .
$$


Proof. We observe that

$$
\begin{aligned}
A T_{1} & =\bigcup_{d \in D_{1}}\left(T_{1}+d\right)=\bigcup_{d \in D_{2}}\left(T_{1}+d-x_{0}\right) \\
& =\bigcup_{d \in D_{2}}\left(T_{1}+d-(A-I)(A-I)^{-1} x_{0}\right) \\
& =\bigcup_{d \in D_{2}}\left(\left(T_{1}+d+(A-I)^{-1} x_{0}\right)\right)-A(A-I)^{-1} x_{0} .
\end{aligned}
$$

By putting the right-most term to the left and by using the uniqueness of the attractor corresponding to $\left(A, D_{2}\right)$, we have $T_{2}=T_{1}+(A-I)^{-1} x_{0}$.

We next consider a case with $A$ replaced by $-A$.

Proposition 2.2. Let $A \in M_{n}(\mathbb{Z})$ be an expanding matrix with $|\operatorname{det} A|=q$, and let $D=\left\{d_{1}, \ldots, d_{q}\right\} \subseteq \mathbb{R}^{n}$. Suppose that there exists a vector $v \in \mathbb{R}^{n}$ such that $D=v-D$. Then $T(-A, D)=T(A, D)-\left(A-A^{-1}\right)^{-1} v$.

Proof. We first consider the case $D=-D$; then

$$
\begin{aligned}
T(-A, D) & =\left\{\sum_{i=1}^{\infty}(-A)^{-i} d_{j_{i}}: d_{j_{i}} \in D\right\} \\
& =\left\{\sum_{i=1}^{\infty} A^{-i}\left((-1)^{i} d_{j_{i}}\right): d_{j_{i}} \in D\right\} \\
& =T(A, D) .
\end{aligned}
$$

For the case $D=v-D$, we let $D^{\prime}=\frac{1}{2} v-D$. Then $D^{\prime}=-D^{\prime}$. A direct application of the above and Proposition 2.1 yields the proposition.

Remark 2.3. From Proposition 2.1, we see that the Lebesgue measure and the connectedness of the attractor $T$ are invariant under a translation of the digit set. Hence we can assume without loss of generality that $0 \in D$ in all the cases. Also, in Proposition 2.2, the condition $D=v-D$ on the digit set $D$ is satisfied in most of the cases in this paper.

Let $A \in M_{n}(\mathbb{Z})$ be expanding with $|\operatorname{det} A|=q$, and let $\Lambda \subseteq \mathbb{R}^{n}$ be a full rank $A$ invariant lattice, that is, $\Lambda$ has rank $n$ and $A(\Lambda) \subseteq \Lambda$. Then the quotient group $\Lambda / A(\Lambda)$ has $q$ distinct cosets. A $q$-digit set $D=\left\{d_{1}, \ldots, d_{q}\right\} \subseteq \Lambda$ is called a complete set of coset representatives of $\Lambda / A(\Lambda)$ if $\Lambda=\bigcup_{i=1}^{q}\left(d_{i}+A(\Lambda)\right)$ and $\left(d_{i}+A(\Lambda)\right) \cap$ $\left(d_{j}+A(\Lambda)\right)=\varnothing$ for $i \neq j$. Using the fact that affine transformations do not affect the property of having positive Lebesgue measure, [1, Theorem 1] can be put in the following form, which we will need later.

TheOREM 2.4. Let $A \in M_{n}(\mathbb{Z})$ be expanding, and let $\Lambda \subseteq \mathbb{R}^{n}$ be a full rank $A$ invariant lattice. Suppose that $D$ is a complete set of coset representatives of $\Lambda / A(\Lambda)$. Then the unique non-empty compact set $T(A, D)$ has positive Lebesgue measure.

It is easy to see that $\mu(T(A, D))>0$ does not imply that $D$ is a complete set of coset representatives. For example, we can take $A=[4]$ and $D=\{0,1,8,9\}$; then we have $T(A, D)=[0,1] \cup[2,3]$. We conclude this section with a proposition on the irreducible polynomials. We use $\mathbb{Q}[x]$ to denote the set of polynomials with rational coefficients, and $\mathbb{Z}[x]$ to denote the set of polynomials with integral coefficients. 
Proposition 2.5. Let $p(x)=x^{m}+a_{m-1} x^{m-1}+\ldots+a_{0}$, where $a_{i} \in \mathbb{Z}$ and $\left|a_{0}\right|=q$ is a prime. Suppose that all the roots $\alpha$ have modulus $>1$; then $p(x)$ is irreducible in $\mathbb{Q}[x]$ and all the roots are simple.

Proof. It is well known that $p(x)$ is irreducible in $\mathbb{Q}[x]$ if and only if it is irreducible in $\mathbb{Z}[x]$ (see, for example, [7, p. 163]). Suppose that $p(x)$ is not irreducible; then $p(x)=g(x) h(x)$ for some non-constant $g(x), h(x) \in \mathbb{Z}[x]$. Since $q$ is a prime, it follows that the constant term of one of the $g(x)$ or $h(x)$ must be \pm 1 , so that at least one of the roots has modulus less than or equal to 1 . This contradicts the hypothesis on $p(x)$, and the irreducibility is proved.

To show that all the roots must be simple, we assume that $p(x)=(x-\alpha)^{\ell} g(x)$ where $\ell \in \mathbb{Z}$, for $\ell>1$. Let

$$
p^{\prime}(x)=\ell(x-\alpha)^{\ell-1} g(x)+(x-\alpha)^{\ell} g^{\prime}(x)
$$

be the formal derivative of $p$. Then $p^{\prime}(x)=m x^{m-1}+(m-1) a_{m-1} x^{m-2}+\ldots+a_{1}$ is in $\mathbb{Z}[x]$ also, and $p(x)$ and $p^{\prime}(x)$ have a common factor $(x-\alpha)$. On the other hand, the division algorithm implies that there exist $s(x), t(x) \in \mathbb{Q}[x]$ such that $p(x) s(x)+$ $p^{\prime}(x) t(x)=g c d \in \mathbb{Q}[x]$. This implies that $g c d(\not \equiv p(x))$ has a factor $(x-\alpha)$, and hence $g c d$ does not equal 1; this contradicts the statement that $p(x)$ is irreducible in $\mathbb{Q}[x]$ just proved.

\section{Integral self-affine tiles}

In the following discussion we consider the collinear digit sets $D=\left\{d_{1} v, \ldots, d_{q} v\right\}$. We let $D^{\prime}=\left\{d_{1}, \ldots, d_{q}\right\}$.

THeorem 3.1. Suppose that $A \in M_{n}(\mathbb{Z})$ is an expanding matrix with $|\operatorname{det} A|=q$, where $q \geqslant 2$ is a prime. Let $D=\left\{d_{1} v, \ldots, d_{q} v\right\}$ with $v \in \mathbb{R}^{n} \backslash\{0\}$, for $d_{i} \in \mathbb{Z}$. Then $T$ is a selfaffine tile (that is, $\mu(T)>0$ ) if and only if $\left\{v, A v, \ldots, A^{n-1} v\right\}$ is a linearly independent set and $D^{\prime}=q^{\prime}\left\{d_{1}^{\prime}, \ldots, d_{q}^{\prime}\right\}$, where $l$ is a nonnegative integer and $\left\{d_{1}^{\prime}, \ldots, d_{q}^{\prime}\right\}$ is a complete set of coset representatives of $\mathbb{Z}_{q}$.

In particular, if $v \in \mathbb{Z}^{n} \backslash\{0\}$, then $\left\{v, A v, \ldots, A^{n-1} v\right\}$ is always a linearly independent set. Hence $T$ is a self-affine tile if and only if the above $\left\{d_{1}^{\prime}, \ldots, d_{q}^{\prime}\right\}$ is a complete set of coset representatives of $\mathbb{Z}_{q}$.

Proof. We first prove the necessity. Since $T=\left\{\sum_{j=1}^{\infty} \beta_{j} A^{-j} v: \beta_{j} \in D^{\prime}\right\}$ and $\mu(T)>0$, it follows that $\left\{v, A v, \ldots, A^{n-1} v\right\}$ is a linearly independent set. To prove the second part, we let $p(x)$ be the characteristic polynomial of $A$, and $C$ be the companion matrix of $p(x)$. $C$ has elements of 1 below the diagonal, the negatives of the coefficients of $p(x)$ in the last column, and zeros at all other entries; it is the matrix representation of the map $A$ with respect to basis $\mathscr{B}=\left\{v, A v, \ldots, A^{n-1} v\right\}$, and $v$ can be represented as $e_{1}=\left[\begin{array}{lll}1 & 0 \ldots 0\end{array}\right]^{t} \in \mathbb{Z}^{n}$. Then $D_{\mathscr{B}}=\left\{d_{1} e_{1}, \ldots, d_{q} e_{1}\right\} \subseteq \mathbb{Z}^{n}$, and $\mu(T(A, D))>0$ if and only if $\mu\left(T\left(C, D_{\mathscr{B}}\right)\right)>0$. Let

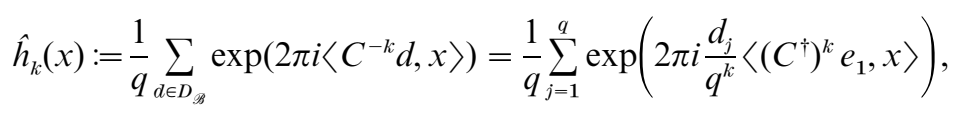

where $C^{\dagger}=q C^{1}$ is an integral matrix. By [10, Theorem 2.1(iii)], $\mu\left(T\left(C, D_{\mathscr{B}}\right)\right)>0$ is equivalent to the following. For each $m \in \mathbb{Z}^{n} \backslash\{0\}$, there exists a nonnegative integer 
$k=k(m)$ such that $\hat{h}_{k}(m)=0$. It follows that $\left\{1 /\left(q^{k-1}\right)\left\langle\left(C^{\dagger}\right)^{k} e_{1}, m\right\rangle d_{j}: d_{j} \in D^{\prime}\right\}$ is a complete set of coset representatives of $\mathbb{Z}_{q}$ for each $m \in \mathbb{Z}^{n} \backslash\{0\}$ (see the proof of [10, Theorem 4.1], assuming that $0 \in D$ by Proposition 2.1). In particular, for $k=k(m)$, we must have

$$
q^{k-1} \mid\left\langle\left(C^{\dagger}\right)^{k} e_{1}, m\right\rangle d_{j} \quad \text { and } \quad q^{k} \chi\left\langle\left(C^{\dagger}\right)^{k} e_{1}, m\right\rangle d_{j}
$$

for each $d_{j} \in D_{\mathscr{B}} \backslash\{0\}$. Thus $D^{\prime}=q^{l}\left\{d_{1}^{\prime}, \ldots, d_{q}^{\prime}\right\}$ with $l$ a nonnegative integer and $\left\{d_{1}^{\prime}, \ldots, d_{q}^{\prime}\right\}$ a complete set of coset representatives of $\mathbb{Z}_{q}$.

To prove the sufficiency, we can assume without loss of generality that $l=0$. Let $\Lambda$ be the lattice generated by the basis $\mathscr{B}=\left\{v, A v, \ldots, A^{n-1} v\right\}$. It is easy to show that $D$ is a complete set of coset representatives of $\Lambda / A(\Lambda)$ and it follows from Theorem 2.4 that $\mu(T)>0$.

To prove the second part, we need only to show that no non-zero vector with rational entries is contained in a proper $A$-invariant subspace; this will imply that $\mathscr{B}$ is a basis of $\mathbb{R}^{n}$. Suppose that $v$ has rational entries and that $\left\{v, A v, \ldots, A^{n-1} v\right\}$ is contained in a proper $A$-invariant subspace of $\mathbb{R}^{n}$; hence it is a linearly dependent set. We have $\sum_{j=0}^{n-1} c_{j} A^{j} v=0$ for some $c_{j} \in \mathbb{R}$, not all zero. Since $A$ and $v$ have rational entries, we can choose $c_{j}$ to be rationals. Let $h(x)=\sum_{j=0}^{n-1} c_{j} x^{j}$; then $h(A) v=0$. It follows that $h(\lambda)=0$ for some eigenvalue $\lambda$ of $A$. This contradicts the assertion that $p(x)$ is irreducible by Proposition 2.5.

COROLlary 3.2. Suppose that $A \in M_{n}(\mathbb{Z})$ is an expanding matrix with $|\operatorname{det} A|=2$. Let $D=\{0, v\}$ for any $v \in \mathbb{Z}^{n} \backslash\{0\}$; then always $\mu(T)>0$.

Proof. We can apply the theorem with $q=2, d_{1}=0, d_{2}=1$.

If $q$ is not assumed to be a prime in Theorem 3.1, then we have the modifications as in the following theorem and in Proposition 3.5.

TheOREM 3.3. Suppose that $A \in M_{n}(\mathbb{Z})$ is an expanding matrix with $|\operatorname{det} A|=q$ (not necessarily prime) and it has $n$ distinct eigenvalues. Let $v=\sum_{i=1}^{n} c_{i} u_{i} \in \mathbb{R}^{n}$, where the $u_{i}$ are the corresponding eigenvectors and $c_{i} \neq 0$ for all $i \in\{1, \ldots, n\}$. Let $D=$ $\left\{d_{1} v, d_{2} v, \ldots, d_{q} v\right\}$ and $D^{\prime}=\left\{d_{1}, d_{2}, \ldots, d_{q}\right\} \subset \mathbb{Z}$. Then $T$ is a self-affine tile (that is, $\mu(T)>0)$ if $D^{\prime}$ is a complete set of coset representatives of $\mathbb{Z}_{q}$.

Moreover, the above $v$ can be chosen to be in $\mathbb{Z}^{n}$.

Proof. We need to show that $\mathscr{B}=\left\{v, A v, \ldots, A^{n-1} v\right\}$ is a basis of $\mathbb{R}^{n}$, and then apply the same idea as in the proof of Theorem 3.1. For $\sum_{i=0}^{n-1} a_{i} A^{i} v$, where $a_{0}, a_{1}, \ldots, a_{n-1} \in \mathbb{R}$ are not all zero, we let $f(x):=a_{0}+a_{1} x+\ldots+a_{n-1} x^{n-1}$; then we can write $\sum_{i=0}^{n-1} a_{i} A^{i} v=\sum_{i=1}^{n} c_{i} f\left(\lambda_{i}\right) u_{i}$ where the $\lambda_{i}$ are the eigenvalues of $A$. Since $f(x)$ can have at most $n-1$ roots and the $\lambda_{i}$ are distinct, we have at least one non-zero $c_{i} f\left(\lambda_{i}\right)$ so that $\sum_{i=0}^{n-1} a_{i} A^{i} v \neq 0$. Hence $\mathscr{B}$ is a linearly independent set, and thus a basis of $\mathbb{R}^{n}$.

To prove the last part, we consider $v=\sum_{i=1}^{n} c_{i} u_{i} \in \mathbb{R}^{n}$ with $c_{i} \in \mathbb{R}$ (or in $\mathbb{C}$ depending on the eigenvectors $u_{i}$ ) in the matrix form $v=U c$. Observe that the set $C_{0}=\left\{c: c_{i}=0\right.$ for some $\left.1 \leqslant i \leqslant n\right\}$ is the union of $n$ (real or complex) hyperplanes and $U^{-1}\left(\mathbb{Z}^{n}\right)$ is a full-rank lattice (in $\mathbb{R}^{n}$ or $\mathbb{C}^{n}$ respectively). Hence $U^{-1}\left(\mathbb{Z}^{n}\right) \nsubseteq C_{0}$ and there exist $v \in \mathbb{Z}^{n}, c \notin C_{0}$ such that $v=U c$, that is, we can find $v=\sum_{i=1}^{n} c_{i} u_{i} \in \mathbb{Z}^{n}$ with $c_{i} \neq 0$. 
We do not have a complete answer on the collinear digit sets when $q$ is not a prime and the eigenvalues of $A$ have higher multiplicities. In $\mathbb{R}^{2}$, we have a substitute for the collinear digit sets (Proposition 3.5 below), which we will use to prove Theorem 1.3. We say that two matrices $A$ and $B$ are $\mathbb{Z}$-similar if there exists a unimodular matrix $P \in G L_{n}(\mathbb{Z})$ such that $P^{-1} A P=B$.

Lemma 3.4. Suppose $A \in M_{2}(\mathbb{Z})$ is an expanding matrix with repeated eigenvalue $\lambda$. Then $\lambda$ is an integer and $A$ is $\mathbb{Z}$-similar to a matrix of the form

$$
\left[\begin{array}{ll}
\lambda & a \\
0 & \lambda
\end{array}\right]
$$

Proof. For such $A$ the characteristic polynomial is $p(x)=(x-\lambda)^{2}$; it is easy to see that $\lambda$ is an integer. Let

$$
v=\left[\begin{array}{l}
x \\
y
\end{array}\right]
$$

be an eigenvector; then $v$ can be chosen as a vector with integral entries $x, y$ such that $\operatorname{gcd}(x, y)=1$. Hence there exist $m, m^{\prime} \in \mathbb{Z}$ such that $x m-y m^{\prime}=1$. Let

$$
P=\left[\begin{array}{cc}
x & m^{\prime} \\
y & m
\end{array}\right] .
$$

Then a direct calculation shows that

$$
P^{-1} A P=\left[\begin{array}{cc}
m & -m^{\prime} \\
-y & x
\end{array}\right] A\left[\begin{array}{cc}
x & m^{\prime} \\
y & m
\end{array}\right]=\left[\begin{array}{ll}
\lambda & a \\
0 & \lambda
\end{array}\right]
$$

for some $a \in \mathbb{Z}$.

Hence for $A$ with repeated eigenvalues, we need only to examine the matrices of the form

$$
A=\left[\begin{array}{ll}
\lambda & a \\
0 & \lambda
\end{array}\right]
$$

If $a=0$, it is obvious that we can take

$$
D=\left\{\left[\begin{array}{l}
i \\
j
\end{array}\right]: 0 \leqslant i, j \leqslant \lambda-1\right\}
$$

and $T(A, D)$ is a square. Otherwise, we have the following proposition.

Proposition 3.5. Let

$$
A=\left[\begin{array}{ll}
\lambda & a \\
0 & \lambda
\end{array}\right], \quad a \neq 0
$$

and let

Then $\mu(T(A, D))>0$.

$$
D=\left\{\left[\begin{array}{c}
i a \\
j
\end{array}\right]: 0 \leqslant i, j \leqslant \lambda-1\right\} \text {. }
$$

Proof. Let $\mathscr{B}=\left\{a e_{1}, e_{2}\right\}$ and let $\Lambda$ be the lattice generated by $\mathscr{B}$; then $\Lambda / A \Lambda$ has $\lambda^{2}$ distinct cosets. In basis $\mathscr{B}$, the matrix of the map $A$ is

$$
\left[\begin{array}{ll}
\lambda & 1 \\
0 & \lambda
\end{array}\right], D=\left\{\left[\begin{array}{l}
i \\
j
\end{array}\right]: 0 \leqslant i, j \leqslant \lambda-1\right\}
$$


and $A(\Lambda)$ contains elements of the form

$$
\left[\begin{array}{c}
\lambda m+m^{\prime} \\
\lambda m^{\prime}
\end{array}\right]
$$

with $m, m^{\prime} \in \mathbb{Z}$. Hence it is easy to see that if $d-d^{\prime} \in A \Lambda$ for

$$
d=\left[\begin{array}{l}
i \\
j
\end{array}\right], \quad d^{\prime}=\left[\begin{array}{l}
i^{\prime} \\
j^{\prime}
\end{array}\right] \in D,
$$

then $\lambda \mid\left(j-j^{\prime}\right)$ and $\lambda \mid\left(i-i^{\prime}\right)$, so that $j=j^{\prime}$ and $i=i^{\prime}$. This implies that $D$ is a complete set of coset representatives of $\Lambda / A(\Lambda)$. We again apply Theorem 2.4 to complete the proof.

\section{A criterion for connectedness}

In [6], it has been shown that if $|\operatorname{det} A|=2$ and $D=\{0, v\} \subseteq \mathbb{Z}^{n}$ is a complete set of coset representatives of the quotient group $\mathbb{Z}^{n} / A \mathbb{Z}^{n}$, then $T(A, D)$ is a connected tile. In fact, we can modify this result as follows.

Proposition 4.1. Suppose that $A \in M_{n}(\mathbb{Z})$ is an expanding matrix with $|\operatorname{det} A|=$ 2. Let $D=\{0, v\}$ such that $v \in \mathbb{R}^{n} \backslash\{0\}$ and $\left\{v, A v, \ldots, A^{n-1} v\right\}$ is a linearly independent set. Then $T(A, D)$ is a pathwise and locally connected tile.

Proof. By Theorem 3.1, we conclude that $T$ is a tile. Also, from the proof of [6], we see that there exists a continuous map from $[0,1]$ onto $T$ (a space filling curve). It follows that $T$ is path-wise and locally connected by the Hahn-Mazurkiewicz Theorem (see [11]).

In the following, we give a general criterion of connectedness by using a 'graph' argument on $D$. Let $(A, D)$ be given; we define

$$
\mathscr{E}=\left\{\left(d_{i}, d_{j}\right):\left(T+d_{i}\right) \cap\left(T+d_{j}\right) \neq \varnothing, d_{i}, d_{j} \in D\right\}
$$

to be the set of 'edges' for the set $D$. We say that $d_{i}$ and $d_{j}$ are $\mathscr{E}$-connected if there exists a finite sequence $\left\{d_{j_{1}}, \ldots, d_{j_{k}}\right\} \subseteq D$ such that $d_{j_{1}}=d_{i}, d_{j_{k}}=d_{j}$ and $\left(d_{j_{l}}, d_{j_{l+1}}\right) \in \mathscr{E}$, $1 \leqslant l \leqslant k-1$. Let $\Delta D:=D-D$.

Proposition 4.2. Let $A \in M_{n}(\mathbb{Z})$ be expanding and let $D \in \mathbb{R}^{n}$ be a digit set. Then $\left(d_{i}, d_{j}\right) \in \mathscr{E}$ if and only if $d_{i}-d_{j}=\sum_{k=1}^{\infty} A^{-k} v_{k}$, for $v_{k} \in \Delta D$.

Proof. Note that for $d_{i}, d_{j}$ in $D,\left(T+d_{i}\right) \cap\left(T+d_{j}\right) \neq \varnothing$ if and only if $d_{i}+$ $\sum_{k=1}^{\infty} A^{-k} d_{j_{k}}=d_{j}+\sum_{k=1}^{\infty} A^{-k} d_{j_{k}}^{\prime}$ for some $\sum_{k=1}^{\infty} A^{-k} d_{j_{k}}$, $\sum_{k=1}^{\infty} A^{-k} d_{j_{k}}^{\prime} \in T$. The proposition follows by setting $v_{k}=d_{j_{k}}^{\prime}-d_{j_{k}}$.

We now give our general criterion for connectedness.

Theorem 4.3. Let $A \in M_{n}(\mathbb{Z})$ be an expanding matrix with $|\operatorname{det} A|=q$, and let $D=\left\{d_{1}, \ldots, d_{q}\right\} \subseteq \mathbb{R}^{n}$ be a q-digit set. Then $T$ is connected if and only if any two $d_{i}, d_{j} \in D$ are $\mathscr{E}$-connected. 
Proof. The necessity is obvious. To prove the sufficiency, we let

$$
S_{j}(x)=A^{-1}\left(x+d_{j}\right), \quad j=1, \ldots, q
$$

and for $J=\left(j_{1}, \ldots, j_{k}\right)$, we let $S_{J}=S_{j_{1}} \circ \ldots \circ S_{j_{k}}$. There exists a large ball $B$ such that $T \subseteq B, S_{j}(B) \subseteq B, j=1,2, \ldots, q$, and

$$
T=\bigcup_{j=1}^{q} S_{j}(T)=\bigcap_{k=1}^{\infty}\left(\bigcup_{|J|=k} S_{J}(B)\right) .
$$

It is clear that $S_{J}(B)$ is also connected. We claim that $\bigcup_{|J|=k} S_{J}(B)$ is connected. For $k=1$, we note that $S_{j}(T) \subseteq S_{j}(B)$ and that the $\mathscr{E}$-connectedness property on $D$ implies that $\bigcup_{j=1}^{q} S_{j}(B)$ is connected. For the inductive step $k+1$, we write

$$
\bigcup_{|J|=k+1} S_{J}(B)=S_{1}\left(\bigcup_{|J|=k} S_{J}(B)\right) \cup \ldots \cup S_{q}\left(\bigcup_{|J|=k} S_{J}(B)\right) \text {. }
$$

By the induction hypothesis, $\bigcup_{|J|=k} S_{J}(B)$ is connected and we can employ the argument above to conclude that $\bigcup_{|J|=k+1} S_{J}(B)$ is connected. This proves the claim.

To show that $T$ is connected, we assume that there exist two components $T_{1}$ and $T_{2}$ such that $\operatorname{dist}\left(T_{1}, T_{2}\right)>\epsilon>0$. Since $\left\{\bigcup_{|J|=k} S_{J}(B)\right\}_{k=1}^{\infty}$ converges to $T$ in the Hausdorff metric, then for $k$ large enough, we have $\operatorname{dist}\left(T, \bigcup_{|J|=k} S_{J}(B)\right)<\epsilon / 4$ and $\operatorname{diam}\left(S_{J}(B)\right)<\epsilon / 4$ for all $|J|=k$. Let

$$
\mathscr{C}_{i}=\left\{J:|J|=k, \operatorname{dist}\left(T_{i}, S_{J}(B)\right)<\frac{\epsilon}{4}\right\}, \quad i=1,2 .
$$

Then from $\operatorname{dist}\left(T_{1}, T_{2}\right)>\epsilon$, we have $\left(\bigcup_{J \in \mathscr{C}_{1}} S_{J}(B)\right) \cap\left(\bigcup_{J \in \mathscr{C}_{2}} S_{J}(B)\right)=\varnothing$. This contradicts the assertion that $\bigcup_{|J|=k} S_{J}(B)$ is connected.

Note that in the above theorem, we do not assume that $\mu(T)>0$. Hence the criterion holds for all attractors $T(A, D)$. For the one-dimensional case, we have the following simple corollary.

Corollary 4.4. Let $q \in \mathbb{Z}$, for $|q| \geqslant 2$. Suppose $A=[q]$ and $D \subseteq \mathbb{R}$ is a $|q|$-digit set. Then $T(A, D)$ is a connected tile if and only if, up to a translation, $D=\{0, a, 2 a, \ldots$, $(|q|-1)$ a\} for some $a>0$.

Proof. The sufficiency is easy: for such $D$, we have $T=[0, a]$ if $q>0$, and by Proposition 2.2, $T=[q a /(|q|+1), a /(|q|+1)]$ if $q<0$.

To prove the necessity, we assume without loss of generality that $q>0$ and $D=$ $\left\{0, d_{1}, \ldots, d_{q-1}\right\}$ with $0<d_{1}<\ldots<d_{q-1}$. Suppose that $D$ is not of the required form; then there exists at least one $d_{j}$ such that $d_{j}-d_{j-1}>\left(d_{q-1}\right) /(q-1)$. If $\left(d_{j}, d_{j-1}\right) \in \mathscr{E}$, then by Proposition 4.2

$$
d_{j}-d_{j-1}=\sum_{k=1}^{\infty} \frac{v_{k}}{q^{k}} \leqslant \sum_{k=1}^{\infty} \frac{d_{q-1}}{q^{k}}=\frac{d_{q-1}}{q-1},
$$

which is a contradiction. Hence $\left(d_{j}, d_{j-1}\right) \notin \mathscr{E}$. In view of the increasing property of the digits, we see that $d_{j}$ and $d_{j-1}$ are not $\mathscr{E}$-connected and $T$ is not connected, by the above theorem. 


\section{More on connectedness}

In this section, we will consider expressions $s_{m}:=\sum_{j=1}^{m} \alpha_{j} A^{m-j} v$, where $\alpha_{j}=0$, $\pm 1, \ldots, \pm(q-1)$. We will call such $s_{m}$ a polynomial for convenience. Also, we define $p_{k}$ to be the shift operator associated with a polynomial $s_{k}^{\prime}=\sum_{j=1}^{k} \alpha_{j}^{\prime} A^{k-j} v$, for $\alpha_{j}^{\prime}=0$, $\pm 1, \ldots, \pm(q-1)$, given by

$$
p_{k}\left(s_{m}\right)=A^{k} s_{m}+s_{k}^{\prime} .
$$

THEOREM 5.1. Let $A \in M_{n}(\mathbb{Z})$ be expanding and let $D=\{0, v, \ldots,(q-1) v\}$ with $v \in \mathbb{R}^{n} \backslash\{0\}$. Then $T(A, D)$ is connected if there exist a monic polynomial $s_{m}$ and a shift operator $p_{k}$ such that $p_{k}\left(s_{m}\right)=s_{m}$.

Proof. To prove the theorem, we need to show that $\left(d_{j}, d_{j+1}\right) \in \mathscr{E}$ for each $j$. By Proposition 4.2 , it suffices to construct a sequence $\left\{\beta_{j}\right\}_{j=0}^{\infty}$ with $\beta_{0}=1$ and $\beta_{j}=0$, $\pm 1, \ldots, \pm(q-1)$ for $j \geqslant 1$ such that $\sum_{j=0}^{\infty} \beta_{j} A^{-j} v=0$. By the hypothesis, we have

$$
\begin{aligned}
p_{k}^{l}\left(s_{m}\right) & =\underbrace{p_{k} \circ p_{k} \circ \ldots \circ p_{k}}_{l \text { times }}\left(s_{m}\right) \\
& =A^{k l} s_{m}+\sum_{j=0}^{l-1} A^{k j} s_{k}^{\prime} \\
& =s_{m} .
\end{aligned}
$$

Let $t_{l}:=A^{-k l}\left(p_{k}^{l}\left(s_{m}\right)\right)=s_{m}+\sum_{j=1}^{l} A^{-k j} s_{k}^{\prime}$ and $t:=\lim _{l \rightarrow \infty} t_{l}$. Then $t=0$ since $t_{l}=A^{-k l} s_{m}$ $\rightarrow 0$. Write $A^{-m+1}(t)=\sum_{j=0}^{\infty} \beta_{j} A^{-j} v$ by inserting the full expressions for $s_{m}$ and $s_{k}^{\prime}$. This series converges since starting from $j=m,\left\{\beta_{j}\right\}_{j=0}^{\infty}$ is periodic (repeated coefficients of $\left.s_{k}^{\prime}\right)$. Also, $\sum_{j=0}^{\infty} \beta_{j} A^{-j} v=0$. We have $\beta_{0}=1$ since $s_{m}$ is monic. Therefore, $\left\{\beta_{j}\right\}_{j=0}^{\infty}$ is the required sequence.

For convenience in the later construction, we include the following corollary.

COROLlaRY 5.2. The shift operator $p_{k}$ in the above theorem can be replaced by $q_{k}$ defined by $q_{k}\left(s_{m}\right)=-A^{k} s_{m}+\sum_{j=1}^{k} \alpha_{j}^{\prime} A^{k-j} v$.

Proof. Suppose there exist $q_{k}$ as above and a monic polynomial $s_{m}$ such that $q_{k}\left(s_{m}\right)=s_{m}$; then we can take $p_{2 k}\left(s_{m}\right)=A^{2 k} s_{m}+s_{2 k}^{\prime}$ with $s_{2 k}^{\prime}=-\sum_{j=1}^{k} \alpha_{j}^{\prime} A^{2 k-j} v+$ $\sum_{j=k+1}^{2 k} \alpha_{j-k}^{\prime} A^{2 k-j} v$, which yields

$$
\begin{aligned}
p_{2 k}\left(s_{m}\right) & =\left(A^{2 k} s_{m}-\sum_{j=1}^{k} \alpha_{j}^{\prime} A^{2 k-j} v\right)+\sum_{j=k+1}^{2 k} \alpha_{j-k}^{\prime} A^{2 k-j} v \\
& =-A^{k}\left(q_{k}\left(s_{m}\right)\right)+\sum_{j=1}^{k} \alpha_{j}^{\prime} A^{k-j} v \\
& =-A^{k} s_{m}+\sum_{j=1}^{k} \alpha_{j}^{\prime} A^{k-j} v \\
& =s_{m} .
\end{aligned}
$$

It follows from Theorem 5.1 that $T(A, D)$ is connected.

The above discussion leads to the following algebraic method to check the connectedness. 
Proposition 5.3. Let $A \in M_{n}(\mathbb{Z})$ be an expanding matrix with $|\operatorname{det} A|=q$ and characteristic polynomial $p(x)$. Let $D=\{0, v, \ldots,(q-1) v\}$ with $v \in \mathbb{R}^{n} \backslash\{0\}$. Suppose that there exists a polynomial $g(x) \in \mathbb{Z}[x]$ such that

$$
h(x)=g(x) p(x)=x^{k}+a_{k-1} x^{k-1}+a_{k-2} x^{k-2}+\ldots+a_{1} x \pm q,
$$

with $\left|a_{i}\right| \leqslant q-1$, for $1 \leqslant i \leqslant k-1$. Then $T(A, D)$ is connected.

Proof. Let $s_{1}=v$. We have two cases.

(i) $h(0)=-q$. We take

$$
p_{k}\left(s_{1}\right)=A^{k} v+\left(a_{k-1} A^{k-1}+a_{k-2} A^{k-2}+\ldots+a_{1} A-(q-1) I\right) v .
$$

Then $p_{k}\left(s_{1}\right)=h(A) v+v=s_{1}$ since $h(A)=0$. By Theorem 5.1, T(A,D) is connected.

(ii) $h(0)=q$. We take

$$
q_{k}\left(s_{1}\right)=-A^{k} v+\left(-a_{k-1} A^{k-1}-a_{k-2} A^{k-2}-\ldots-a_{1} A-(q-1) I\right) v,
$$

so that $q_{k}\left(s_{1}\right)=-h(A) v+v=s_{1}$. In this case, Corollary 5.2 implies that $T(A, D)$ is connected.

We need the following lemma for the characteristic polynomial of $A \in M_{2}(\mathbb{Z})$ which can easily be proved by using the quadratic formula [2].

Lemma 5.4. Let $f(x)=x^{2}+a x \pm q$, where $a \in \mathbb{Z}, 1 \neq q \in \mathbb{N}$. Then $f(x)$ is expanding if and only if $|a| \leqslant q$ for $f(0)=q$, and $|a| \leqslant q-2$ for $f(0)=-q$.

We can now apply Proposition 5.3 to conclude the following theorem.

TheOREM 5.5. Let $A \in M_{2}(\mathbb{Z})$ be an expanding matrix with $|\operatorname{det} A|=q$ (not necessarily prime). Then there exists a digit set $D=\left\{d_{1}, \ldots, d_{q}\right\} \subseteq \mathbb{Z}^{2}$ such that $T$ is a connected tile.

Proof. That $T$ is a tile follows from Theorem 3.3 and Proposition 3.5 for an appropriate choice of $D$. To show the connectedness of $T$, we let $p(x)=x^{2}+a x \pm q$ be the characteristic polynomial of $A$. We divide our consideration into two cases.

Case (i): $A$ has simple eigenvalues. By Theorem 3.3 , we can take $D=\{0, v, \ldots$, $(q-1) v$ \} for some $v$. By Lemma 5.4, we see that all the characteristic polynomials $p(x)$ satisfy the condition in Proposition 5.3 with $g(x) \equiv 1$, except for the case $p(x)=$ $x^{2}+q x+q$. For that, we let $g(x)=x-1$; then

$$
h(x)=(x-1) p(x)=(x-1)\left(x^{2}+q x+q\right)=x^{3}+(q-1) x^{2}-q
$$

again satisfies the condition in Proposition 5.3. Hence $T$ is connected.

Case (ii): $A$ has a repeated eigenvalue $\lambda$. We cannot apply Proposition 5.3 because the digit set $D$ given in Proposition 3.5 is not a collinear digit set. By Lemma 3.4, we only need to examine the matrices of the form

$$
A=\left[\begin{array}{ll}
\lambda & a \\
0 & \lambda
\end{array}\right]
$$

If $a=0$, then we choose

$$
D=\left\{\left[\begin{array}{l}
i \\
j
\end{array}\right]: 0 \leqslant i, j \leqslant \lambda-1\right\}
$$


as our digit set. Then $T(A, D)=[0,1]^{2}$ is a connected tile.

If $a \neq 0$, we take

$$
D=\left\{\left[\begin{array}{c}
i a \\
j
\end{array}\right]: 0 \leqslant i, j \leqslant \lambda-1\right\}
$$

as in Proposition 3.5. We observe that

It follows that

$$
\sum_{j=1}^{\infty} A^{-j}\left[\begin{array}{l}
a \\
0
\end{array}\right]=(A-I)^{-1}\left[\begin{array}{l}
a \\
0
\end{array}\right]=\frac{1}{(\lambda-1)}\left[\begin{array}{l}
a \\
0
\end{array}\right] .
$$

$$
\left[\begin{array}{c}
(\alpha+1) a \\
0
\end{array}\right]-\left[\begin{array}{c}
\alpha a \\
0
\end{array}\right]=\left[\begin{array}{l}
a \\
0
\end{array}\right]=\sum_{j=1}^{\infty} A^{-j}\left[\begin{array}{c}
(\lambda-1) a \\
0
\end{array}\right]
$$

for $0 \leqslant \alpha \leqslant \lambda-2$ and

$$
\left[\begin{array}{c}
\beta a \\
\gamma
\end{array}\right]-\left[\begin{array}{c}
\beta a \\
\gamma-1
\end{array}\right]=\left[\begin{array}{l}
0 \\
1
\end{array}\right]=\sum_{j=1}^{\infty} A^{-j}\left[\begin{array}{c}
a \\
\lambda-1
\end{array}\right]
$$

for $0 \leqslant \beta \leqslant \lambda-1,1 \leqslant \gamma \leqslant \lambda-1$. Then Proposition 4.2 and Theorem 4.3 imply that $T(A, D)$ is connected.

In the rest of this section, we apply the criterion in Proposition 5.3 to consider some special connected tiles in $\mathbb{R}^{3}$. The following lemma, combined with Proposition 2.2 , enables us to consider only the characteristic polynomials with positive constant terms, that is, $\operatorname{det} A<0$.

Lemma 5.6. Let $p(x)$ and $\tilde{p}(x)$ denote the characteristic polynomials of $A,-A \in$ $M_{n}(\mathbb{Z})$ respectively. Then $\tilde{p}(x)=(-1)^{n} p(-x)$.

Proposition 5.7. Let $A \in M_{3}(\mathbb{Z})$ be expanding with $|\operatorname{det} A|=q=3$, 4 or 5 ; then there exists a q-digit set $D$ such that $T(A, D)$ is a self-affine connected tile.

Proof. We first consider the case with $\operatorname{det} A=-3$ in $\mathbb{R}^{3}$. For $D=\{0, v, 2 v\}$ as in Theorem 3.1, the corresponding $T$ is a tile. We use Proposition 5.3 to check the connectedness. There are 25 expanding characteristic polynomials $p(x)$ with $p(0)=3 ; 17$ of them are already in the form of $h(x)$ in Proposition 5.3. The rest of them can be reduced to the form of $h(x)$ by multiplying a polynomial $g(x)$ as shown in Table 1.

TABle 1. $g(x)$ for the case $\operatorname{det} A=3$.

\begin{tabular}{ccc}
\hline Characteristic polynomial $=p(x)$ & $g(x)$ & $h(x)=g(x) p(x)$ \\
\hline$x^{3}-3 x+3$ & $\left(x^{2}+1\right)(x+1)$ & $x^{6}+x^{5}-2 x^{4}+x^{3}+3$ \\
$x^{3}+x^{2}-4 x+3$ & $\left(x^{2}+1\right)(x+1)$ & $x^{6}+2 x^{5}-2 x^{4}+x^{3}-x+3$ \\
$x^{3}+x^{2}-3 x+3$ & $x+1$ & $x^{4}+2 x^{3}-2 x^{2}+3$ \\
$x^{3}+2 x^{2}+3 x+3$ & $x-1$ & $x^{4}+x^{3}+x^{2}-3$ \\
$x^{3}+3 x^{2}+2 x+3$ & $x-1$ & $x^{4}+2 x^{3}-x^{2}+x-3$ \\
$x^{3}+3 x^{2}+3 x+3$ & $x-1$ & $x^{4}+2 x^{3}-3$ \\
$x^{3}+3 x^{2}+4 x+3$ & $x-1$ & $x^{4}+2 x^{3}+x^{2}-x-3$ \\
$x^{3}+4 x^{2}+5 x+3$ & $\left(x^{2}-x+1\right)(x-1)$ & $x^{6}+2 x^{5}-x^{4}+x-3$ \\
\hline
\end{tabular}

For the case $\operatorname{det} A=-4$, a direct check shows that all the characteristic polynomials have simple eigenvalues; hence Theorem 3.3 implies that for a suitable 
$v, D=\{0, v, 2 v, 3 v\}$ will give a tile $T$. To check the connectedness, we see that 35 of the $p(x)$ are already in the form of $h(x)$ in Proposition 5.3. The rest can also be shown to satisfy such form by the multiplication of suitable polynomials $g(x)$. (There are 16 of them; see Table 2.)

TABLE 2. $g(x)$ for the case $\operatorname{det} A=4$.

\begin{tabular}{ccc}
\hline Characteristic polynomial $=p(x)$ & $g(x)$ & $h(x)=g(x) p(x)$ \\
\hline$x^{3}-4 x+4$ & $\left(x^{2}+1\right)(x+1)$ & $x^{6}+x^{5}-3 x^{4}+x^{3}+4$ \\
$x^{3}+x^{2}-4 x+4$ & $x+1$ & $x^{4}+2 x^{3}-3 x^{2}+4$ \\
$x^{3}+x^{2}-5 x+4$ & $\left(x^{2}+1\right)(x+1)$ & $x^{6}+2 x^{5}-3 x^{4}+x^{3}-x+4$ \\
$x^{3}+2 x^{2}-6 x+4$ & $\left(x^{2}+1\right)(x+1)$ & $x^{6}+3 x^{5}-3 x^{4}+x^{3}-2 x+4$ \\
$x^{3}+2 x^{2}-5 x+4$ & $x+1$ & $x^{4}+3 x^{3}-3 x^{2}-x+4$ \\
$x^{3}+2 x^{2}-4 x+4$ & $x+1$ & $x^{4}+3 x^{3}-2 x^{2}+4$ \\
$x^{3}+2 x^{2}+4 x+4$ & $x-1$ & $x^{4}+x^{3}+2 x^{2}-4$ \\
$x^{3}+3 x^{2}+4 x+4$ & $x-1$ & $x^{4}+2 x^{3}+x^{2}-4$ \\
$x^{3}+3 x^{2}+5 x+4$ & $x-1$ & $x^{4}+2 x^{3}+2 x^{2}-x-4$ \\
$x^{3}+4 x^{2}+2 x+4$ & $x-1$ & $x^{4}+3 x^{3}-2 x^{2}+2 x-4$ \\
$x^{3}+4 x^{2}+3 x+4$ & $x-1$ & $x^{4}+3 x^{3}-x^{2}+x-4$ \\
$x^{3}+4 x^{2}+4 x+4$ & $x-1$ & $x^{4}+3 x^{3}-4$ \\
$x^{3}+4 x^{2}+5 x+4$ & $x-1$ & $x^{4}+3 x^{3}+x^{2}-x-4$ \\
$x^{3}+4 x^{2}+6 x+4$ & $x-1$ & $x^{4}+3 x^{3}+2 x^{2}-2 x-4$ \\
$x^{3}+5 x^{2}+6 x+4$ & $(x-1)^{2}$ & $x^{5}+3 x^{4}-3 x^{3}-3 x^{2}-2 x+4$ \\
$x^{3}+5 x^{2}+7 x+4$ & $\left(x^{2}+1\right)(x-1)^{2}$ & $x^{7}+3 x^{6}-x^{5}-2 x^{4}-3 x^{3}-x^{2}-x+4$ \\
\hline
\end{tabular}

For the case $\operatorname{det} A=-5$, we basically go through the same process as in the case $\operatorname{det} A=-3$. There are 85 expanding characteristic polynomials with constant coefficient $5 ; 26$ of them are non-trivial, as shown in Table 3 .

TABle 3. $g(x)$ for the case $\operatorname{det} A=5$.

\begin{tabular}{ccc}
\hline Characteristic polynomial $=p(x)$ & $g(x)$ & $h(x)=g(x) p(x)$ \\
\hline$x^{3}-5 x+5$ & $\left(x^{2}+1\right)(x+1)$ & $x^{6}+x^{5}-4 x^{4}+x^{3}+5$ \\
$x^{3}+x^{2}-5 x+5$ & $x+1$ & $x^{4}+2 x^{3}-4 x^{2}+5$ \\
$x^{3}+x^{2}-6 x+5$ & $\left(x^{2}+1\right)(x+1)$ & $x^{6}+2 x^{5}-4 x^{4}+x^{3}-x+5$ \\
$x^{3}+2 x^{2}+5 x+5$ & $x-1$ & $x^{4}+x^{3}+3 x^{2}-5$ \\
$x^{3}+2 x^{2}-5 x+5$ & $x+1$ & $x^{4}+3 x^{3}-3 x^{2}+5$ \\
$x^{3}+2 x^{2}-6 x+5$ & $x+1$ & $x^{4}+3 x^{3}-4 x^{2}-x+5$ \\
$x^{3}+2 x^{2}-7 x+5$ & $\left(x^{2}+1\right)(x+1)$ & $x^{6}+3 x^{5}-4 x^{4}+x^{3}-2 x+5$ \\
$x^{3}+3 x^{2}+6 x+5$ & $x-1$ & $x^{4}+2 x^{3}+3 x^{2}-x-5$ \\
$x^{3}+3 x^{2}+5 x+5$ & $x-1$ & $x^{4}+2 x^{3}+2 x^{2}-5$ \\
$x^{3}+3 x^{2}-5 x+5$ & $x+1$ & $x^{4}+4 x^{3}-2 x^{2}+5$ \\
$x^{3}+3 x^{2}-6 x+5$ & $x+1$ & $x^{4}+4 x^{3}-3 x^{2}-x+5$ \\
$x^{3}+3 x^{2}-7 x+5$ & $x+1$ & $x^{4}+4 x^{3}-4 x^{2}-2 x+5$ \\
$x^{3}+3 x^{2}-8 x+5$ & $\left(x^{2}+1\right)(x+1)$ & $x^{6}+4 x^{5}-4 x^{4}+x^{3}-3 x+5$ \\
$x^{3}+4 x^{2}+7 x+5$ & $x-1$ & $x^{4}+3 x^{3}+3 x^{2}-2 x-5$ \\
$x^{3}+4 x^{2}+6 x+5$ & $x-1$ & $x^{4}+3 x^{3}+2 x^{2}-x-5$ \\
$x^{3}+4 x^{2}+5 x+5$ & $x-1$ & $x^{4}+3 x^{3}+x^{2}-5$ \\
$x^{3}+5 x^{2}+8 x+5$ & $x-1$ & $x^{4}+4 x^{3}+3 x^{2}-3 x-5$ \\
$x^{3}+5 x^{2}+7 x+5$ & $x-1$ & $x^{4}+4 x^{3}+2 x^{2}-2 x-5$ \\
$x^{3}+5 x^{2}+6 x+5$ & $x-1$ & $x^{4}+4 x^{3}+x^{2}-x-5$ \\
$x^{3}+5 x^{2}+5 x+5$ & $x-1$ & $x^{4}+4 x^{3}-5$ \\
$x^{3}+5 x^{2}+4 x+5$ & $x-1$ & $x^{4}+4 x^{3}-x^{2}+x-5$ \\
$x^{3}+5 x^{2}+3 x+5$ & $x-1$ & $x^{4}+4 x^{3}-2 x^{2}+2 x-5$ \\
$x^{3}+5 x^{2}+2 x+5$ & $x-1$ & $x^{4}+4 x^{3}-3 x^{2}+3 x-5$ \\
$x^{3}+6 x^{2}+9 x+5$ & $\left(x^{2}+1\right)(x-1)^{2}$ & $x^{7}+4 x^{6}-x^{5}-3 x^{4}-3 x^{3}-2 x^{2}-x+5$ \\
$x^{3}+6 x^{2}+8 x+5$ & $(x-1)^{2}\left(x^{2}+1\right)\left(x^{3}+1\right)$ & $x^{10}+4 x^{9}-2 x^{8}-x^{6}-2 x^{5}-3 x^{4}-2 x+5$ \\
$x^{3}+6 x^{2}+7 x+5$ & $(x-1)^{2}$ & $x^{5}+4 x^{4}-4 x^{3}-3 x^{2}-3 x+5$ \\
\hline
\end{tabular}




\section{Remarks}

We have seen in the last section that in many cases the characteristic polynomial can be reduced to the form in Proposition 5.3 so that the connectedness holds. We do not know whether this will hold in general. With regard to this, we give the following result of Garsia [4, Lemma 1.6] (modified into the present setting).

If $A \in M_{n}(\mathbb{Z})$ is an expanding matrix with $|\operatorname{det} A|=q$ (not necessarily prime), and if $\lambda$ is a root of the characteristic polynomial of $A$, then $\lambda$ satisfies an integral polynomial with coefficients $0, \pm 1, \ldots, \pm q$.

There is another interesting question related to connectedness: the determination of whether the tile is disk-like. Some partial results can be found in [2]. They show that in $\mathbb{R}^{2}$, for $A \in M_{2}(\mathbb{Z})$ with $|\operatorname{det} A|=3$, some of the connected tiles obtained with collinear digit sets are not disk-like. In fact, there is no disk-like tile for $A$ with det $A=-3$ and $\operatorname{tr}(A)= \pm 1$. We do not know much about this in general. However, in $\mathbb{R}^{2}$, we have the following partial result.

Proposition 6.1. Suppose that $A \in M_{2}(\mathbb{Z})$ is an expanding matrix with $|\operatorname{det} A|=q$ and characteristic polynomial $p(x)=x^{2} \pm q$. Then there exists a q-digit set $D=$ $\left\{d_{1}, \ldots, d_{q}\right\} \subseteq \mathbb{Z}^{2}$ such that $T(A, D)$ is a disk-like tile.

Proof. Let

$$
A=\left[\begin{array}{ll}
a_{1} & a_{2} \\
a_{3} & a_{4}
\end{array}\right] .
$$

We first assume that $a_{2} \neq 0$; then we have $a_{4}=-a_{1}, a_{3}=-\left(a_{1}^{2} \pm q\right) / a_{2}$.

Case (i). If $|\operatorname{det} A|=q$ is an odd number, let

$$
v=\left[\begin{array}{l}
0 \\
1
\end{array}\right] \text { and } D=\{0, \pm v, \ldots, \pm q-1 / 2 v\} \text {. }
$$

Then $T(A, D)=\left\{\left(x,-a_{1} / a_{2} x+t\right):|x| \leqslant\left|a_{2}\right| / 2,|t| \leqslant \frac{1}{2}\right\}$ is a parallelogram.

Case (ii). If $|\operatorname{det} A|=q$ is an even number, then we let

and

$$
v=\left[\begin{array}{c}
0 \\
-1
\end{array}\right], D=\{v, \ldots, q v\} \text { if } \operatorname{det} A=q,
$$

$$
v=\left[\begin{array}{l}
0 \\
1
\end{array}\right], D=\{0, v, \ldots,(q-1) v\} \text { if } \operatorname{det} A=-q .
$$

Then it follows from direct calculation that

$$
T(A, D)= \begin{cases}\left\{\left(x,-\frac{a_{1}}{a_{2}} x+t\right): 0 \leqslant x \leqslant a_{2}, 0 \leqslant t \leqslant 1\right\} & \text { if } a_{2}>0, \\ \left\{\left(x,-\frac{a_{1}}{a_{2}} x+t\right): a_{2} \leqslant x \leqslant 0,0 \leqslant t \leqslant 1\right\} & \text { if } a_{2}<0\end{cases}
$$

is again a parallelogram.

It remains to consider the case $a_{2}=0$. Let

$$
P=\left[\begin{array}{cc}
1 & -1 \\
0 & 1
\end{array}\right] \text { if } a_{3}=0 \text { and } P=\left[\begin{array}{ll}
0 & 1 \\
1 & 0
\end{array}\right] \text { if } a_{3} \neq 0 .
$$


Let $D^{\prime}=P D$, where $D$ is as in cases (i), (ii), and $A^{\prime}=P^{-1} A P$. Then $T\left(A, D^{\prime}\right)=$ $P T\left(A^{\prime}, D\right)$, and $T\left(A^{\prime}, D\right)$ is again a parallelogram.

The proof can easily be implemented on Matlab by checking the coordinates of the corner points.

Acknowledgements. The authors would like to thank Professor Y. Wang for introducing this topic while he was visiting the Institute of Mathematical Sciences at the Chinese University of Hong Kong. They would also like to thank the referee for many helpful suggestions and comments about revising the paper. Thanks are also extended to Professor K. W. Leung for clarifying some algebraic facts, and to Dr S. M. Ngai and Dr H. Rao for modifying the connectedness criterion. The first author was supported by a grant from Sakarya University in Turkey and the Institute of Mathematical Sciences at the Chinese University of Hong Kong. The second author was supported by an RGC grant from Hong Kong.

Note added in proof, April 2000. We have proved that Proposition 5.7 is true for any expanding $A \in M_{3}(\mathbb{Z})$. The detail will appear elsewhere.

\section{References}

1. C. BANDT, 'Self-similar sets. 5: Integer matrices and fractal tilings of $\mathbb{R}^{n}$ ', Proc. Amer. Math. Soc. 112 (1991) 549-562.

2. C. BANDT and G. Gelbrich, 'Classification of self-affine lattice tilings', J. London Math. Soc. 50 (1994) 581-593.

3. K. J. FALCONER, Fractal geometry: mathematical foundations and applications (John Wiley, 1990).

4. A. Garsia, 'Arithmetic properties of Bernoulli convolutions', Trans. Amer. Math. Soc. 102 (1962) $409-432$.

5. K. Gröchenig and A. HaAs, 'Self-similar lattice tilings', J. Fourier Anal. Appl. 1 (1994) 131-170.

6. D. Hacon, N. C. Saldanha and J. J. P. Veerman, 'Remarks on self-affine tilings', Experiment. Math. 3 (1994) 317-327.

7. T. W. Hungerford, Algebra, 2nd edn (Springer, New York, 1996).

8. J. E. Hutchinson, 'Fractals and self-similarity', Indiana Univ. Math. J. 30 (1981) 713-747.

9. J. C. Lagarias and Y. Wang, 'Self-affine tiles in $\mathbb{R}^{n}$, Adv. Math. 121 (1996) 21-49.

10. J. C. LAGarias and Y. WANG, 'Integral self-affine tiles in $\mathbb{R}^{n}$. I: Standard and nonstandard digits sets', J. London Math. Soc. 54 (1996) 161-179.

11. S. Willard, General topology (Addison-Wesley, 1970).

Department of Mathematics

University of Pittsburgh

Pittsburgh

$P$ A 15260

USA

ibkst+@pitt.edu
Department of Mathematics Chinese University of Hong Kong

Hong Kong

kslau@math.cuhk.edu.hk 